\title{
Moyamoya Disease: Epidemiology, Clinical Features, and Diagnosis
}

\author{
Jong S. Kim \\ Stroke Center and Department of Neurology, Asan Medical Center, University of Ulsan, Seoul, Korea
}

Moyamoya disease (MMD) is a chronic, occlusive cerebrovascular disease characterized by progressive stenosis at the terminal portion of the internal carotid artery and an abnormal vascular network at the base of the brain. Although its etiology remains unknown, recent genetic studies identified RNF213 in the 17q25-ter region as an important susceptibility gene of MMD among East Asian populations. Possibly because of genetic differences, MMD is relatively common in people living in East Asian countries such as Korea and Japan, compared to those in the Western Hemisphere. The prevalence of MMD appears to be slightly lower among Chinese, compared to Koreans or Japanese. There are two peaks of incidence with different clinical presentations, at around 10 years and 30-40 years. The peak appears to occur later in women than men. In children, ischemic symptoms, especially transient ischemic attacks, are predominant. Intellectual decline, seizures, and involuntary movements are also more common in this age group. In contrast, adult patients present with intracranial hemorrhage more often than pediatric patients. In patients with MMD, intracerebral hemorrhage is more often accompanied by intraventricular hemorrhage than in patients with hypertensive intracerebral hemorrhage. These different age peaks and different clinical presentations in each age group are also observed in MMD patients in the USA. Catheter angiography is the diagnostic method of choice. Magnetic resonance (MR) angiography and computed tomographic angiography are noninvasive diagnostic methods. High-resolution vessel wall MR imaging also helps diagnose MMD by revealing concentric vessel wall narrowing with basal collaterals.

Keywords Moyamoya disease; Epidemiology; Clinical features; Diagnosis

\author{
Correspondence: Jong S. Kim \\ Department of Neurology, Asan Medical \\ Center, University of Ulsan College of \\ Medicine, 88 Olympic-ro 43-gil, \\ Songpa-gu, Seoul 05505, Korea \\ Tel: +82-2-3010-3440 \\ Fax: +82-2-474-4691 \\ E-mail: jongskim@amc.seoul.kr
}

Received: November 12, 2015 Revised: December 24, 2015 Accepted: December 28, 2015

This study was supported by a grant from the Ministry for Health, Welfare and Family Affairs, Republic of Korea (HI14C1985).

The authors have no financial conflicts of interest.

\section{Introduction}

Moyamoya disease (MMD), also called spontaneous occlusion of the circle of Willis, is a chronic, occlusive cerebrovascular disease of unknown etiology characterized by steno-occlusive changes at the terminal portion of the internal carotid artery (ICA) and an abnormal vascular network at the base of the brain. ${ }^{1}$ In light of the increasing number of patients with unilateral involvement ${ }^{2}$ as well as evidence that a substantial number of unilateral cases progress to bilateral presentation $, 3,4$ the diagnostic criteria for definitive MMD were revised to include patients with both bilateral and unilateral presentation of terminal ICA stenosis with an abnormal vascular network at the base of the brain (statement by the Research Committee of MMD of the Japanese Ministry of Health, Labour, and Welfare in 2015). Diagnostic criteria also state that definitive diagnosis of MMD requires catheter angiography in unilateral cases, whereas bilateral cases can be promptly diag- 
nosed by either catheter angiography or magnetic resonance imaging/angiography (MRI/MRA). When there are causative diseases or associated conditions, terms such as moyamoya syndrome and angiographic moyamoya are often used. ${ }^{5}$ Although uncommon, MMD is an important cause of nonatherosclerotic intracranial arterial disease, especially in East Asian countries. In these regions, isolated middle cerebral artery stenosis is seen in young patients, with eventual evolution into MMD. ${ }^{6} \mathrm{MMD}$ is the most important cause of stroke or transient ischemic attack (TIA) in children in this part of the world. Here, the epidemiology, clinical features, and diagnosis of MMD are reviewed.

\section{Epidemiology}

For an unknown reason, $\mathrm{MMD}$ is relatively common in people living in East Asian countries such as Korea and Japan, as compared to those in the Western Hemisphere. Table 1 summarizes epidemiological study results in East Asian countries. According to a survey performed in Japan in 1995, the prevalence of MMD was approximately 3.16/100,000, with an estimated incidence of $0.35 / 100,000 .^{7}$ A subsequent survey in 2004 showed that the number of patients considerably increased, with an estimated incidence of $0.54 / 100,000$, and prevalence of 6.03/100,000 in 2003. ${ }^{8}$ Another study performed in Hokkaido, Japan, ${ }^{8}$ showed that the incidence and prevalence were $0.94 / 100,000$ and 10.5/100,000, respectively, based on 267 newly diagnosed MMD patients between 2002 and 2006. The male-to-female ratio was 1:1.8 or 1:2.2 in these surveys, and approximately $10 \%-15 \%$ of patients had a family history. The risk of having MMD in family members is about 30-40 times higher than the general population., ${ }^{7,9}$ There were two peaks of incidence, at 10-20 and 35-50 years old.

An epidemiology study from Korea based on National Health Insurance (NHI) data ${ }^{10}$ reported that the prevalence of MMD increased from 6.3/100,000 in 2004 to 9.1/100,000 in 2008. The incidence was $1.0 / 100,000$ in 2008. Beginning in 2006, the NHI initiated a registration program for 138 rare diseases, including MMD. Patients registered in this program become eligible for a copayment reduction. According to a study using the data set obtained from 2007 to $2011,{ }^{11}$ the number of incident MMD cases increased from 848 to 1,192 , with the annual incidence being 1.7 to $2.3 / 100,000$. In both studies, the female-to-male ratio was 1.9 , and there were two peaks of incidence; the peak in the pediatric group occurred at age 5 to 14 years, accounting for $16.2 \%$ of all incident cases, and the peak in the adult group occurred at age 45 to 54 years, accounting for $22.8 \%{ }^{11}$ (Figure 1). The overall prevalence of
MMD in 2011 was as high as 16.1/100,000.

Although Korea has an apparently higher incidence and prevalence of MMD compared with Japan, it should be noted that a direct comparison is difficult for the following reasons. There were differences in data collection methods and population characteristics; Japanese studies used data from hospital surveys, with a limited response rate. On the other hand, Korean studies were based on national insurance data. Moreover, because registered patients were financially supported from 2006, there is a possibility of overdiagnosis. Additionally, the Japanese study analyzed data in 1995 and 2006, whereas the Korean studies analyzed data from 2004 to 2011 . Thus, a progression of time may be the reason for the apparently higher prevalence in Korea.

Recently, the inpatient databases of the Taiwan NHI program identified MMD patients during 2000 to $2011 .{ }^{12}$ During the 12 -year period, 422 patients were identified, representing an annual incidence of $0.15 / 100,000$ person-years. Adults exhibited an upward trend, with an incidence rate of 1.74 in 2010 to 2011 compared with 2000 to 2001 . Thus, the incidence of MMD has increased in adults but not in children from 2000 to 2011 in Taiwan. For unknown reasons, compared with patients hospitalized during 2000 to 2005 , patients identified during 2006 to 2011 had a greater female-to-male ratio (1.7 vs. 1.1). Epidemiology studies in mainland China are rare. An epidemiological study in the area of Nanjing, the capital city of Jiangsu province, with a total population of approximately 6.2 million, showed that the prevalence was $3.92 / 100,000$ during 2000-2007, ${ }^{13}$ which was generally lower than that in Korea or Japan (Table 1). Unlike series from Korea and Japan, hemorrhage was more common than ischemic stroke in this cohort, probably due to the fact that the second peak (adult group) was larger than the first (pediatric group). However, this study was based on data from a small part of China. A more recent Chinese study on 802 MMD patients ${ }^{14}$ showed that clinical presentations are similar to those from Korea and Japan. In both studies, age distribution of the patients with MMD had a bimodal occurrence: at age 5 to 9 years, and 35 to 39 years. Although reliable, national epidemiologic studies on mainland China are not yet available; given the lower incidence of MMD in the Taiwan study based on NHI data, the incidence of MMD in Chinese people may be lower than in Korean and Japanese counterparts. More surprisingly, unlike Korea and Japan, there is no difference in sex distribution of MMD in Chinese patients. ${ }^{13,14}$ However, these studies do not represent data from all of China. Moreover, the female-to-male ratio increased over time from 1.1 (2000 to 2005) to 1.7 (2006-2011) in a Taiwan study. ${ }^{12}$ Therefore, a 


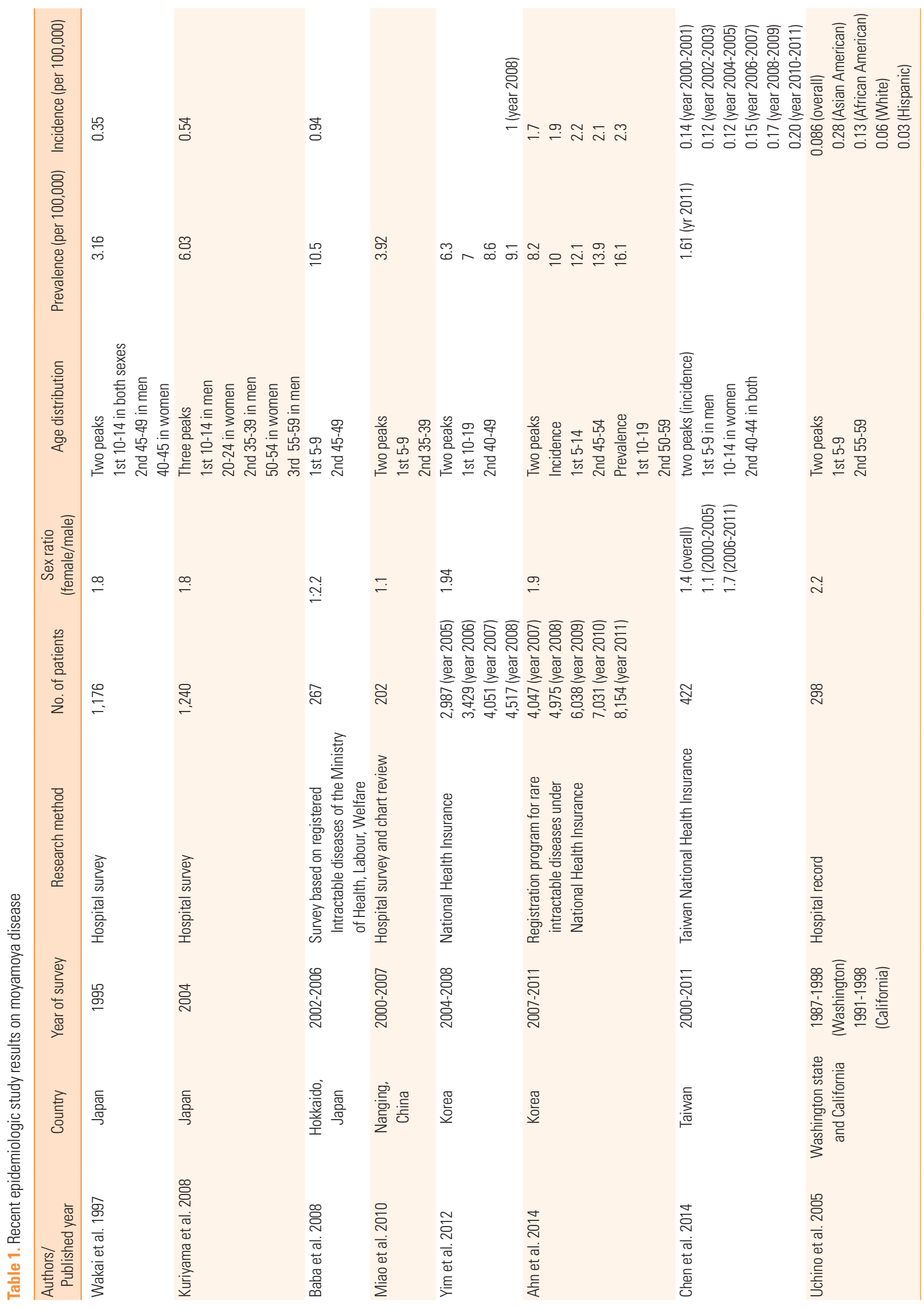



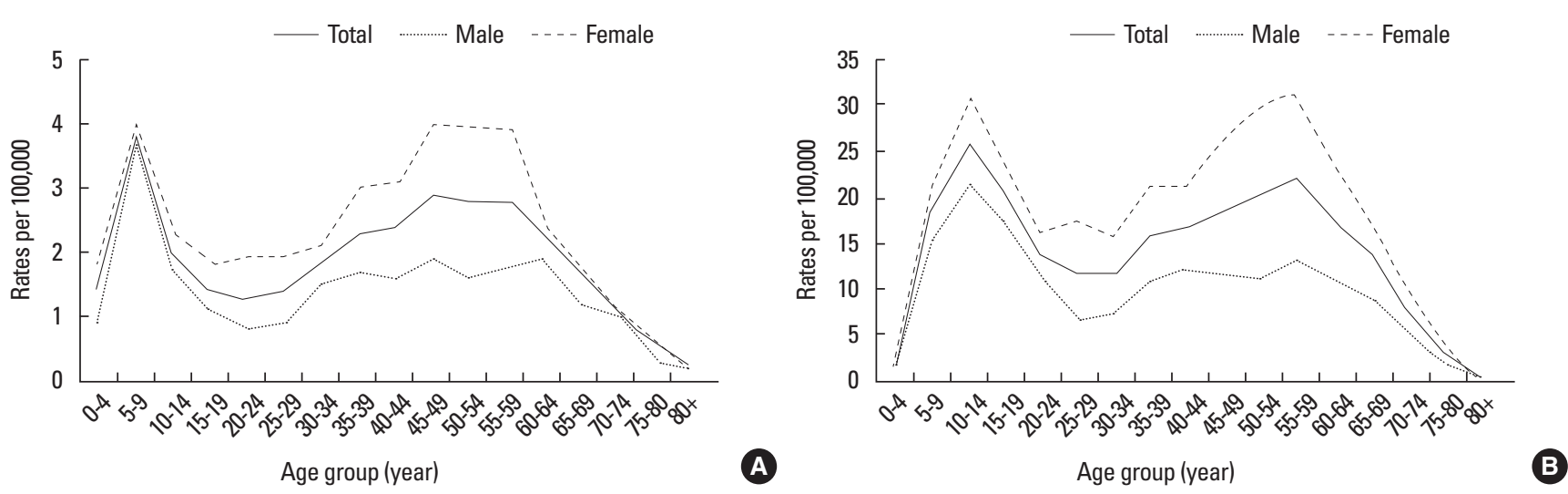

Figure 1. Bimodal age distribution in moyamoya disease patients (From: Ahn IM, et al. Incidence, Prevalence, and Survival of Moyamoya Disease in Korea: A Nationwide, Population-Based Study. Stroke 2014;45:1090-1095). (A) Incidence of moyamoya disease by sex and age in Korea, 2007 to 2011. (B) Prevalence of moyamoya disease by sex and age in Korea, 2011.

conclusion should be cautiously drawn. In addition, familial occurrence of MMD has been variably reported: $1.5 \%{ }^{13}$ or $5.2 \%$ in China, ${ }^{14}$ and $10 \%-15 \%$ in Japan. ${ }^{7,8}$ Although familial cases were previously (in 1997) reported to be low (2\%) in South Korea, ${ }^{15}$ more recent studies showed that familial history was found in $10 \%-15 \%$ of MMD patients. ${ }^{16-18}$ The ratio of familial cases may change according to the extensiveness of diagnostic workup; a recent Chinese study ${ }^{19}$ screened 285 immediate family members of 245 sporadic MMD patients with transcranial Doppler (TCD) and identified 41 cases of familial MMD. Thus, the familial cases increased from 7\% (before screening) to $15 \%$ (after screening). Because TCD has a high diagnostic agreement with MRA, the authors recommended implementation of TCD screening in all family members of MMD patients to detect asymptomatic cases. As shown in Table 1, there is an increasing incidence and prevalence of MMD over time in East Asian countries. This finding may indicate an actual increase in the incidence of MMD in these countries. However, a more plausible explanation would be an increase in newly diagnosed patients owing to the recent advent of noninvasive diagnostic tools such as MRA. Another explanation would be an increasing number of survivors due to improved management. Studies from outside of Asia are rare. In Washington state and California, the incidence of MMD was reported to be $0.086 / 100,000$ based on 298 patients. The incidence was the highest in Asians, followed by Blacks, Whites, and Hispanics (Table 1). The incidence in Asian Americans was 4.6 times higher than that in Whites. Female preponderance was also noted. ${ }^{20}$ African-Americans had an earlier disease onset with a median age of 18 . However, a more recent study based on the Nationwide Inpatient Sample database reported that MMD appears to be distributed among the races according to their relative proportions in the
USA population. ${ }^{21}$ From 2005 to 2008, there were an estimated 7,473 (2,236 pediatric and 5,237 adult) patients admitted with a diagnosis of MMD in the USA. MMD patients were most frequently Caucasians. Overall, ischemic stroke was the most common reason for admission in both children and adults. Hemorrhagic stroke was more frequent in adults compared with children, and there was a bimodal age distribution with peaks in the first and fourth decades of life. Female-tomale ratio was 2.2. Thus, MMD in the USA does not seem to differ from East Asian MMD.

\section{Clinical features}

The clinical presentations of MMD include TIA, ischemic stroke, hemorrhagic stroke, seizures, headache, and cognitive impairment. The incidence of each symptom varies according to the age of the patients. ${ }^{22}$ An ischemic event is the most important clinical manifestation of MMD. Cerebral hypoperfusion due to progressive major vessel occlusion results in repeated hemodynamic TIAs or ischemic strokes in children or young adults. Thus, MMD is a classic example of a hemodynamic cause of stroke. We often encounter patients who develop repeated TIAs when they are hyperventilating, crying, eating hot noodles, or playing a harmonica or flute. Decreased arterial $\mathrm{PaCO}_{2}$ due to hyperventilation may possibly induce vasodilation of normal vessels and subsequent hypoperfusion in vulnerable areas via a steal phenomenon. Stress, fatigue, infection, and dehydration may also precipitate ischemic symptoms. Less often, patients have territorial infarctions due to embolism or thrombotic occlusion in the distribution of the middle cerebral artery (MCA), anterior cerebral artery (ACA), or posterior cerebral artery (PCA) (Figures 2 and 3$){ }^{23}$ The anterior circulation is predominantly involved, and the most 

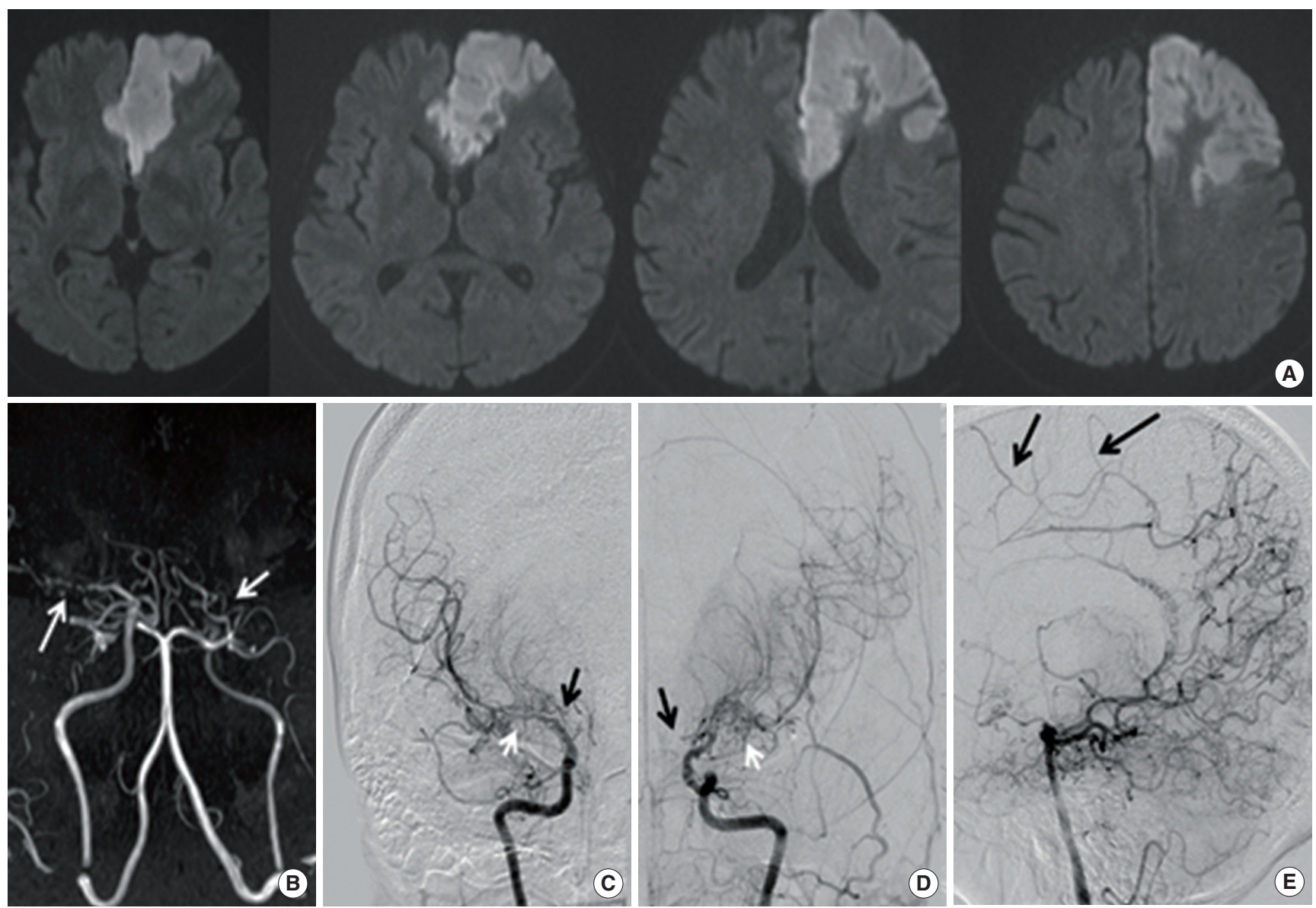

Figure 2. A 47-year-old woman developed abulia, apathy, and somnolence. Diffusion-weighted MRI showed left ACA territory infarction. The infarcted area expanded slightly to MCA territory (A). MRA showed diffuse stenosis in the left MCA and right distal internal carotid artery occlusion (arrows, B). Transfemoral angiography showed bilateral MCA steno-occlusion (white arrows), ACA occlusion (black arrows), and basal collaterals (C,D). Well-developed posterior cerebral arteries supplying the convexity area (arrows) explain why this area was spared after ACA occlusion (E).

frequent ischemic symptom is hemiparesis, followed by speech disturbances and hemisensory abnormalities. ${ }^{24}$ In one study that examined 410 pediatric MMD cases, ischemic symptoms were attributed to MCA territory in 92, ACA territory in 52, and PCA territory in $10 .{ }^{25}$ Although posterior circulation stroke has been considered uncommon, developing in the late stage of MMD, ${ }^{26}$ a more recent study reported that PCA involvement was present in $29 \%$ of patients, with $17 \%$ demonstrating PCA territory infarction. ${ }^{27}$ Unexpectedly, the prevalence of PCA involvement was not much different (26\% vs. $33 \%$ ) between pediatric and adult patients. Although PCA involvement is the natural disease process of $\mathrm{MMD}$, given the observation that PCA disease progression frequently occurs after anterior circulation revascularization procedures, some argue that secondary stenosis (involution of the PCA) may also occur, owing to improved collaterals to the anterior circulation. ${ }^{28}$ Regardless of the cause, PCA involvement is one of the factors related to poor prognosis in MMD. ${ }^{25}$ Recently, a correlation between the homozygous c.14576G > A variant of the ring finger protein 213 and early onset and aggressive PCA in volvement has been shown, ${ }^{29}$ providing further evidence for the significance of PCA stenosis as a prognostic factor. In MMD patients, the infarct topography does not often fit in classic vascular territory (Figures 2 and 3). This is partly because of the concomitant presence of hemodynamic insufficiency and partly because of altered vascular territories secondary to long-standing major vessel occlusion, along with diversely developed collateral channels. In one study, ${ }^{30}$ the authors examined diffusion-weighted MRI patterns of 91 cerebral infarcts from $66 \mathrm{MMD}$ patients. Each ischemic lesion was categorized as an unusual type (gyral, atypical territorial, and honeycomb patterns) or a typical (usually seen in stroke patients) type (territorial, multiple-dot, borderzone, and deep lacunar pattern). The infarct pattern was more often atypical (gyral, 44\%, atypical territorial 13\%, and honeycomb 11\%) than typical (territorial 10\%, multiple-dot $9 \%$, borderzone 6 $\%$, and deep lacunar $8 \%)$. Gyral and borderzone patterns were more frequently seen in pediatric ( $<20$ years old) MMD pa- 

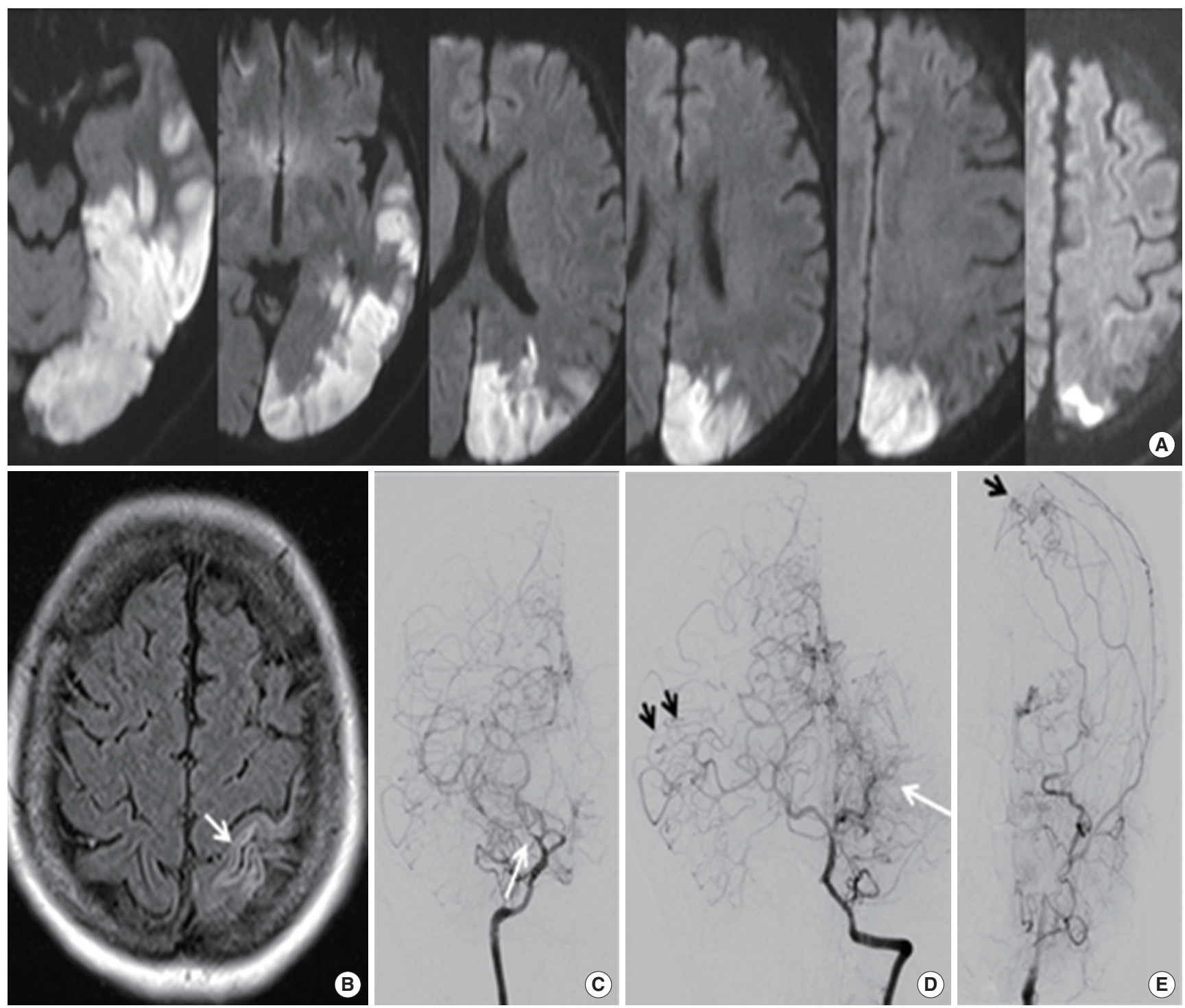

Figure 3. A 44-year-old woman developed transient right limb weakness. Six months later, she developed headache and right homonymous hemianopia. Diffusion-weighted MRI showed an infarction in the left PCA territory (A). FLAIR MRI (B) showed an old infarction in the left parietal area explaining previous hemiparesis. Transfemoral angiography showed an occlusion of the right MCA with basal collaterals (arrow, C). The left PCA was occluded with development of fine collaterals (white arrow). The right PCA was intact and supplied the right MCA territory (dark arrow, D). There was an occlusion of the left distal internal carotid artery (not shown) and part of the left MCA territory was supplied by a prominent external carotid artery system (arrow, E)

tients, whereas a honeycomb pattern was more common in adult patients with advanced vascular stages, which may be associated with age-specific vulnerability of the brain to ischemia, stage of arteriopathy, or changes of abnormal collateral pathways. Approximately $30 \%$ of MMD patients present with intracerebral hemorrhage ( $\mathrm{ICH}$ ) (or less commonly, subarachnoid hemorrhage) secondary to friable collateral vessels harboring microaneurysms or false aneurysms. ${ }^{24}$ The hemorrhages usually occur in the anterior circulation territory. Although any brain region can be affected, intraventricular hemorrhage occurs more commonly than in hypertensive hemorrhage. ${ }^{31}$ In one case-control study that compared 93 patients with $\mathrm{ICH}$ associated with $\mathrm{MMD}$ and those with primary $\mathrm{ICH},{ }^{32}$ the location of hemorrhage was significantly different between the two groups (Figure 4). The order of frequency of the location was putaminal (46.2\%), thalamic (19.4\%), pontine (14\%), lobar (9.7\%), cerebellar (4.3\%), caudate (4.3\%), and pure intraventricular $(2.2 \%)$ in primary ICH, compared to intraventricular (37.6\%), lobar (23.7\%), and putaminal (22.6\%) in MMD patients. ICH in the pons and cerebellum only occurred in primary ICH patients, whereas callosal hemorrhage $(4.3 \%)$ only occurred in MMD-associated ICH. Primary intraventricular hemorrhage in MMD patients was closely associated with a prominent ipsilateral anterior choroidal artery, suggesting that 

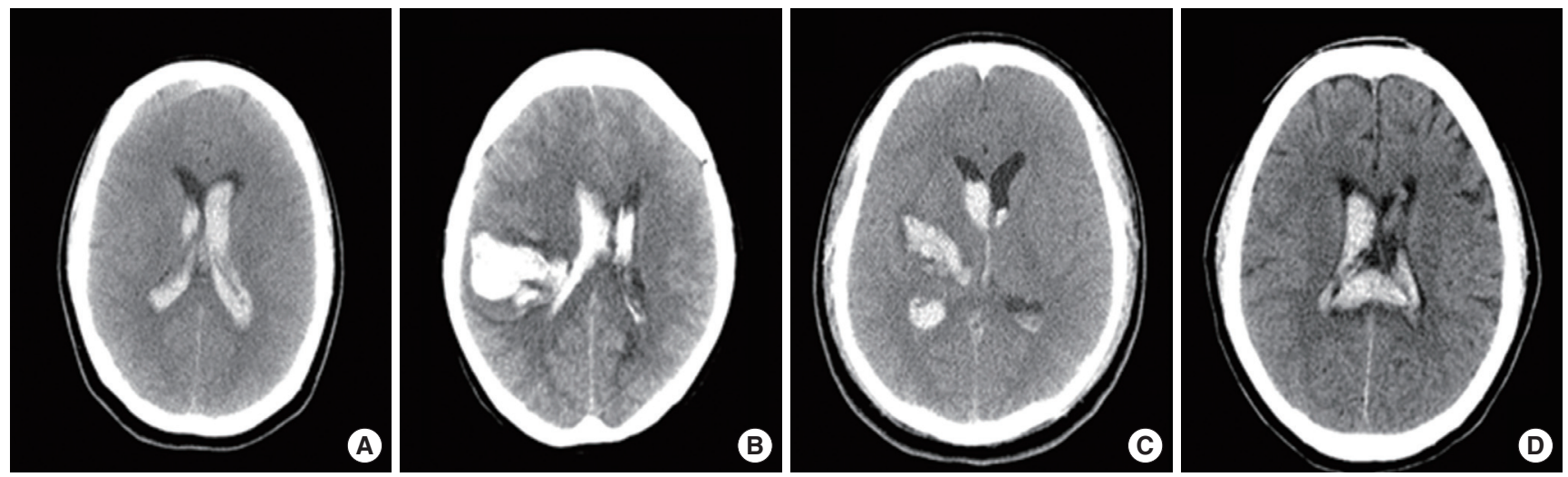

Figure 4. Representative patients with moyamoya disease-related hemorrhage. (A) Primary IVH; (B) lobar hemorrhage with IVH; (C) putaminal hemorrhage with IVH; (D) callosal hemorrhage with IVH. IVH, intraventricular hemorrhage. (From: Nah HW, et al. Moyamoya Disease-Related Versus Primary Intracerebral Hemorrhage: Location and Outcomes Are Different. Stroke 2012;43:1947-1950).

rupture of focal microaneurysms in the abnormally dilated anterior choroidal artery branches may produce intraventricular hemorrhages. In addition, it has been shown that gradientecho T2* weighted or susceptibility-weighted MRI identify cerebral microbleeds (CMBs) in $28 \%-46 \%$ of MMD patients. ${ }^{33-35}$ According to Sun et al., ${ }^{35} \mathrm{CMBs}$ most often occurred in deep and periventricular areas, and are associated with dilated anterior choroidal or posterior communicating arteries. They hypothesized that CMBs represent red blood cells leaked from these dilated, fragile vessels. Periventricular CMBs were associated with future occurrence of intraventricular hemorrhages. Thus, MMD should be suspected when the patients are young, without vascular risk factors, or when the location of the hemorrhage is atypical. For an unclear reason, hemorrhagic strokes are less common in Caucasians than in Asians. ${ }^{31,36,37}$

With significant brain hypoperfusion, cognitive impairment, intellectual decline, or mental retardation may develop, ${ }^{38-40}$ which is a grave problem for children of school age. Seizures occur in approximately $5 \%$ of patients secondary to an ischemic lesion or hypoperfusion, usually starting in childhood. Headache either presents as a symptom of MMD or newly develops after bypass surgery. Although the prevalence of headache in MMD has been considered to be low, ${ }^{24}$ a recent study ${ }^{41}$ reported that 44 of 204 (21.6\%) children with MMD suffered from headache, and nausea/vomiting were seen in 12. In 4, headache developed during hyperventilation, and in 3, TIA and headache occurred simultaneously. The cause of headache in MMD remains unclear. Cerebral hypoperfusion may possibly lower the threshold for migraine development and increase the risk of spreading cortical depression. ${ }^{42,43}$ The headache occasionally improves after the revascularization process, suggesting that cerebral hypoperfusion is related to the pathogenesis of headache at least in some of these patients. The experi- ence of preoperative headache is a strong predictor of postoperative headache. ${ }^{41}$ Although uncommon, MMD may present with involuntary movements, usually in children ${ }^{44}$; in one study, 17 of 410 (4\%) pediatric MMD patients developed involuntary movements. ${ }^{25}$ Baik and Lee ${ }^{45}$ studied 42 patients (38 described in previous studies and 4 from their hospital) who developed involuntary movements associated with MMD. Twenty-seven patients developed chorea; 4, dystonia; 4, a mixture of both; and 7, dyskinesia. In 37 patients, brain imaging studies showed ischemic lesions, but the remaining 5 showed no parenchymal lesions. Studies showed cerebral hypoperfusion in the basal ganglia and in the cerebral cortical areas on the relevant side. In most of the patients, symptoms improved regardless of treatment.

There are two peaks of incidence with different clinical presentations, at around 10 years and 30-40 years, although one Japanese study reported that there are three peaks. ${ }^{9}$ The peak appears to occur later in women than in men. ${ }^{9}$ In children, ischemic symptoms, especially TIAs, are predominant (70\%). ${ }^{25,46}$ Intellectual decline, seizures, and involuntary movements are also more common in this age group. In contrast, adult patients present with intracranial hemorrhage more often than pediatric patients. These different age peaks and clinical presentations in each age group were also observed in MMD patients living in the USA. ${ }^{20,21}$ There have been debates about whether MMD in European Caucasians differs from that in Asians. A recent study on 153 European Caucasians with $\mathrm{MMD}^{47}$ showed that although they also have two age peaks (11-18 and 40-49 years), the later peak was longer than in Asian patients. The later peak was associated with a stronger female preponderance (2.9:1). On the other hand, only $1.3 \%$ of cases were familial. Despite a relatively predominant adult patient population, hemorrhagic stroke overall occurred less frequently (8.5\%) than in Asian 

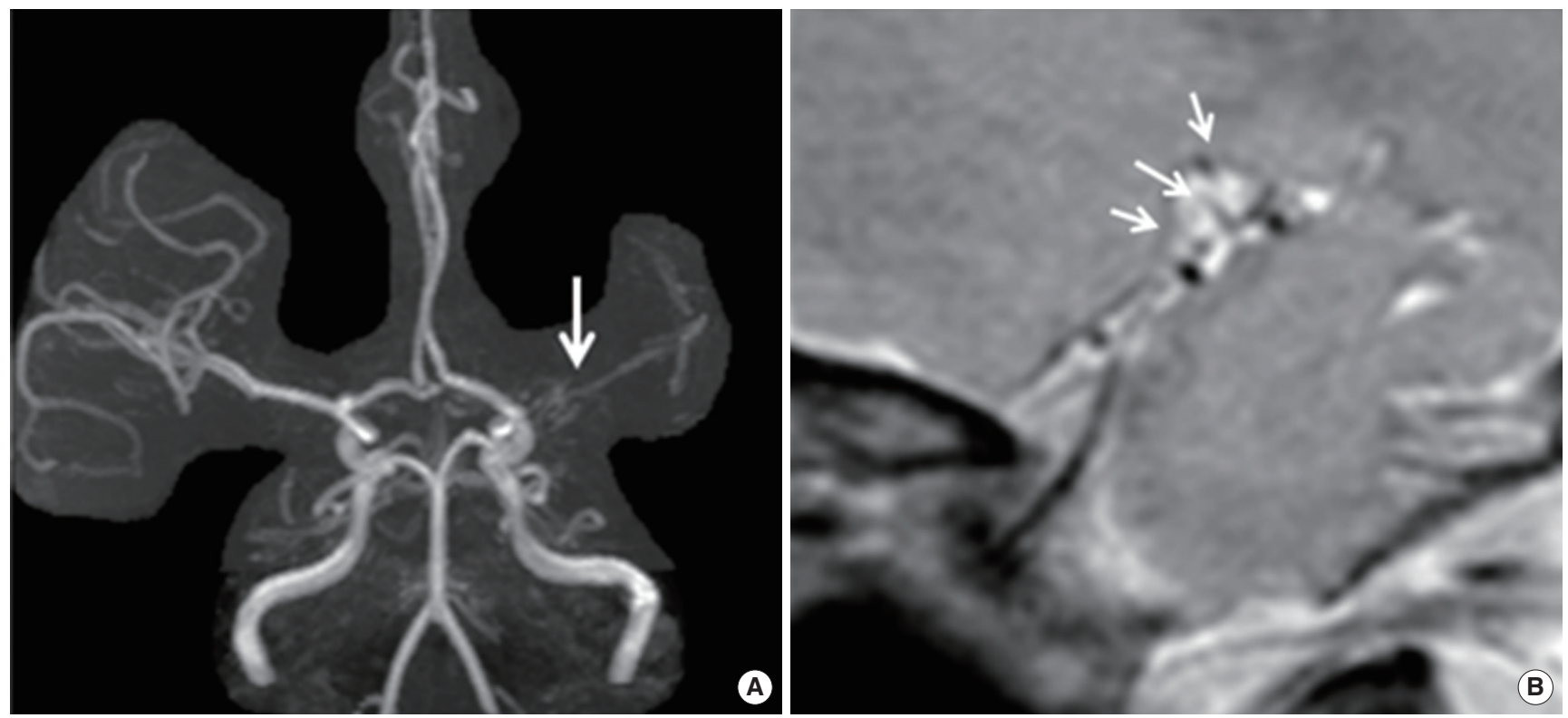

Figure 5. A 33-year-old woman developed transient right sided weakness MRA showed unilateral MCA stenosis (arrow, A). High-resolution vessel wall MRI showed concentric narrowing of vessels with basal collaterals (arrows, B). RNF213 polymorphism was positive, further supporting the diagnosis of moyamoya disease.

patients, and occurred more frequently in pediatric (12\%) than adult (7.8\%) patients. However, this should be interpreted cautiously, because this was not a national survey and the majority were preselected surgical patients.

\section{Diagnosis}

Diagnostic criteria previously required that steno-occlusive change at the ICA should be evident bilaterally for the definitive diagnosis of MMD. Recently, however, diagnostic criteria for definitive MMD were revised to include patients with unilateral terminal ICA steno-occlusion as well. Definitive diagnosis of MMD requires catheter angiography in unilateral cases, while bilateral cases can be promptly diagnosed by either catheter angiography or MRA (Figures 2 and 3). Although catheter angiography is the most important diagnostic test, this relatively invasive technique is associated with procedure-related complications, and therefore preprocedural measures including sufficient hydration should be appropriately taken, especially in pediatric patients. Based on various angiographic findings, Suzuki and Takaku ${ }^{1}$ proposed 6 stages of angiographic evolution. However, a stepwise progression from stage 1 through stage 6 has been observed in only a limited number of patients, ${ }^{42}$ and the practical value of the classification remains unclear. MRA and computed tomography angiography are noninvasive tests that can detect distal ICA or MCA steno-occlusion. However, they have limited sensitivity in detecting basal collaterals. Modern supportive diagnostic tools are useful in making an accurate diagnosis of MMD, especially in patients in an early stage of Suzuki's angiographic grading system, ${ }^{1}$ when an abnormal vascular network is not yet evident. To improve diagnostic capability, high resolution vessel wall MRI has recently been used. Kaku and colleagues proposed a constrictive remodeling theory in which outer diameter narrowing of the affected intracranial vessels was an early characteristic change of MMD, as demonstrated by three-dimensional (3D) constructive interference in steady-state (CIISS) MRI. ${ }^{48}$ Yuan et al. also reported that vascular wall thinning and arterial outer diameter narrowing shown by high resolution MRI could be early morphological changes characteristic of MMD. ${ }^{49}$ Besides outer diameter narrowing, concentric enhancement of symptomatic segments was observed in MMD patients. ${ }^{50}$ Thus, high-resolution MRI including 3D-CIISS could provide supportive information for the accurate diagnosis of MMD, especially in the early angiographic stage. In addition, genetic analysis as described in another review would provide supportive information for the diagnosis of MMD (Figure 5).

\section{Summary}

Recent epidemiological studies have confirmed that MMD is most common in East Asian countries such as Korea and Japan. Although the prevalence of MMD appears to be slightly lower among the Chinese, larger national epidemiological studies are still needed in China. In East Asian countries, clinical features of MMD are similar; there are two peaks of inci- 
dence, at around 10 years and at 30-40 years, with different clinical presentations. In children, ischemic symptoms, especially TIA, are predominant, whereas intracranial hemorrhage is more common in adult patients. In Asian countries, the incidence of MMD appears to increase over time. Patients with MMD are less frequently described in the West, and there seems to be a lower incidence of familial cases. These differences may be attributed to the more significant role of MMD genetic susceptibility in East Asians than in Caucasians. However, reliable studies are still needed to understand the epidemiology, pathophysiology, and genetics of MMD.

\section{References}

1. Suzuki J, Takaku A. Cerebrovascular "moyamoya” disease. Disease showing abnormal net-like vessels in base of brain. Arch Neurol 1969;20:288-299.

2. Hayashi K, Horie N, Izumo T, Nagata I. A nationwide survey on unilateral moyamoya disease in Japan. Clin Neurol Neurosurg 2014;124:1-5.

3. Kelly ME, Bell-Stephens TE, Marks MP, Do HM, Steinberg GK. Progression of unilateral moyamoya disease: a clinical series. Cerebrovasc Dis 2006;22:109-115.

4. Kuroda S, Ishikawa T, Houkin K, Nanba R, Hokari M, Iwasaki $\mathrm{Y}$. Incidence and clinical features of disease progression in adult moyamoya disease. Stroke 2005;36:2148-2153.

5. Natori Y, Ikezaki K, Matsushima T, Fukui M. 'Angiographic moyamoya' its definition, classification, and therapy. Clin Neurol Neurosurg 1997;99 Suppl 2:S168-172.

6. Choi HY, Lee JE, Jung YH, Cho HJ, Kim DJ, Heo JH. Progression of isolated middle cerebral artery stenosis into moyamoya disease. Neurology 2007;68:954.

7. Wakai K, Tamakoshi A, Ikezaki K, Fukui M, Kawamura T, Aoki R, et al. Epidemiological features of moyamoya disease in Japan: Findings from a nationwide survey. Clin Neurol Neurosurg 1997;99 Suppl 2:S1-5.

8. Baba T, Houkin K, Kuroda S. Novel epidemiological features of moyamoya disease. J Neurol Neurosurg Psychiatry 2008;79: 900-904.

9. Kuriyama S, Kusaka Y, Fujimura M, Wakai K, Tamakoshi A, Hashimoto S, et al. Prevalence and clinicoepidemiological features of moyamoya disease in Japan: findings from a nationwide epidemiological survey. Stroke 2008;39:42-47.

10. Im SH, Cho CB, Joo WI, Chough CK, Park HK, Lee KJ, et al. Prevalence and epidemiological features of moyamoya disease in Korea. J Cerebrovasc Endovasc Neurosurg 2012;14:75-78.

11. Ahn IM, Park DH, Hann HJ, Kim KH, Kim HJ, Ahn HS. Incidence, prevalence, and survival of moyamoya disease in Korea: a nationwide, population-based study. Stroke 2014;45:10901095.

12. Chen PC, Yang SH, Chien KL, Tsai IJ, Kuo MF. Epidemiology of moyamoya disease in Taiwan: a nationwide populationbased study. Stroke 2014;45:1258-1263.

13. Miao W, Zhao PL, Zhang YS, Liu HY, Chang Y, Ma J, et al. Epidemiological and clinical features of moyamoya disease in Nanjing, China. Clin Neurol Neurosurg 2010;112:199-203.

14. Duan L, Bao XY, Yang WZ, Shi WC, Li DS, Zhang ZS, et al. Moyamoya disease in China: its clinical features and outcomes. Stroke 2012;43:56-60.

15. Ikezaki K, Han DH, Kawano T, Kinukawa N, Fukui M. A clinical comparison of definite moyamoya disease between South Korea and Japan. Stroke 1997;28:2513-2517.

16. Yeon JY, Shin HJ, Kong DS, Seol HJ, Kim JS, Hong SC, et al. The prediction of contralateral progression in children and adolescents with unilateral moyamoya disease. Stroke 2011;42: 2973-2976.

17. Cho WS, Kim JE, Kim CH, Ban SP, Kang HS, Son YJ, et al. Long-term outcomes after combined revascularization surgery in adult moyamoya disease. Stroke 2014;45:3025-3031.

18. Jo KI, Yeon JY, Hong SC, Kim JS. Clinical course of asymptomatic adult moyamoya disease. Cerebrovasc Dis 2014;37:94-101.

19. Han C, Feng H, Han YQ, Liu WW, Zhang ZS, Yang WZ, et al. Prospective screening of family members with moyamoya disease patients. PLoS One 2014;9:e88765.

20. Uchino K, Johnston SC, Becker KJ, Tirschwell DL. Moyamoya disease in Washington state and California. Neurology 2005;65: 956-958

21. Kainth D, Chaudhry SA, Kainth H, Suri FK, Qureshi AI. Epidemiological and clinical features of moyamoya disease in the USA. Neuroepidemiology 2013;40:282-287.

22. Research Committee on the Pathology and Treatment of Spontaneous Occlusion of the Circle of Willis; Health Labour Sciences Research Grant for Research on Measures for Intractable Diseases. Guidelines for diagnosis and treatment of moyamoya disease (spontaneous occlusion of the circle of Willis). Neurol Med Chir (Tokyo) 2012;52:245-266.

23. Horn P, Bueltmann E, Buch CV, Schmiedek P. Arterio-embolic ischemic stroke in children with moyamoya disease. Childs Nerv Syst 2005;21:104-107.

24. Suzuki J, Kodama N. Moyamoya disease--a review. Stroke 1983; 14:104-109.

25. Kim SK, Cho BK, Phi JH, Lee JY, Chae JH, Kim KJ, et al. Pediatric moyamoya disease: An analysis of 410 consecutive cases. Ann Neurol 2010;68:92-101.

26. Kim JM, Lee SH, Roh JK. Changing ischaemic lesion patterns in adult moyamoya disease. J Neurol Neurosurg Psychiatry 2009; 
80:36-40.

27. Hishikawa T, Tokunaga K, Sugiu K, Date I. Assessment of the difference in posterior circulation involvement between pediatric and adult patients with moyamoya disease. J Neurosurg 2013;119:961-965.

28. Lee JY, Kim SK, Phi JH, Wang KC. Posterior cerebral artery insufficiency in pediatric moyamoya disease. J Korean Neurosurg Soc 2015;57:436-439.

29. Miyatake S, Miyake N, Touho H, Nishimura-Tadaki A, Kondo Y, Okada I, et al. Homozygous c.14576g >a variant of $\operatorname{rnf} 213$ predicts early-onset and severe form of moyamoya disease. Neurology 2012;78:803-810.

30. Cho HJ, Jung YH, Kim YD, Nam HS, Kim DS, Heo JH. The different infarct patterns between adulthood-onset and childhood-onset moyamoya disease. J Neurol Neurosurg Psychiatry 2011;82:38-40.

31. Chiu D, Shedden P, Bratina P, Grotta JC. Clinical features of moyamoya disease in the United States. Stroke 1998;29:13471351.

32. Nah HW, Kwon SU, Kang DW, Ahn JS, Kwun BD, Kim JS. Moyamoya disease-related versus primary intracerebral hemorrhage: location and outcomes are different. Stroke 2012;43: 1947-1950.

33. Kikuta K, Takagi Y, Nozaki K, Sawamoto N, Fukuyama H, Hashimoto N. The presence of multiple microbleeds as a predictor of subsequent cerebral hemorrhage in patients with moyamoya disease. Neurosurgery 2008;62:104-111.

34. Mori N, Miki Y, Kikuta K, Fushimi Y, Okada T, Urayama S, et al. Microbleeds in moyamoya disease: susceptibility-weighted imaging versus $\mathrm{t} 2{ }^{*}$-weighted imaging at 3 tesla. Invest Radiol 2008;43:574-579.

35. Sun W, Yuan C, Liu W, Li Y, Huang Z, Zhu W, et al. Asymptomatic cerebral microbleeds in adult patients with moyamoya disease: A prospective cohort study with 2 years of follow-up. Cerebrovasc Dis 2013;35:469-475.

36. Yilmaz EY, Pritz MB, Bruno A, Lopez-Yunez A, Biller J. Moyamoya: Indiana University Medical Center experience. Arch Neurol 2001;58:1274-1278.

37. Hallemeier CL, Rich KM, Grubb RL Jr, Chicoine MR, Moran CJ, Cross DT 3rd, et al. Clinical features and outcome in North American adults with moyamoya phenomenon. Stroke 2006;37:1490-1496.

38. Ikezaki K, Matsushima T, Kuwabara Y, Suzuki SO, Nomura T, Fukui M. Cerebral circulation and oxygen metabolism in child- hood moyamoya disease: A perioperative positron emission tomography study. J Neurosurg 1994;81:843-850.

39. Hogan AM, Kirkham FJ, Isaacs EB, Wade AM, Vargha-Khadem F. Intellectual decline in children with moyamoya and sickle cell anaemia. Dev Med Child Neurol 2005;47:824-829.

40. Imaizumi C, Imaizumi T, Osawa M, Fukuyama Y, Takeshita M. Serial intelligence test scores in pediatric moyamoya disease. Neuropediatrics 1999;30:294-299.

41. Seol HJ, Wang KC, Kim SK, Hwang YS, Kim KJ, Cho BK. Headache in pediatric moyamoya disease: review of 204 consecutive cases. J Neurosurg 2005; 103:439-442.

42. Olesen J, Friberg L, Olsen TS, Andersen AR, Lassen NA, Hansen $\mathrm{PE}$, et al. Ischaemia-induced (symptomatic) migraine attacks may be more frequent than migraine-induced ischaemic insults. Brain 1993;116:187-202.

43. Park-Matsumoto YC, Tazawa T, Shimizu J. Migraine with auralike headache associated with moyamoya disease. Acta Neurol Scand 1999;100:119-121.

44. Kuroda S, Houkin K. Moyamoya disease: current concepts and future perspectives. Lancet Neurol 2008; 7:1056-1066.

45. Baik JS, Lee MS. Movement disorders associated with moyamoya disease: A report of 4 new cases and a review of literatures. Mov Disord 2010;25:1482-1486.

46. Kim SK, Seol HJ, Cho BK, Hwang YS, Lee DS, Wang KC. Moyamoya disease among young patients: its aggressive clinical course and the role of active surgical treatment. Neurosurgery 2004;54:840-844.

47. Acker G, Goerdes S, Schneider UC, Schmiedek P, Czabanka M, Vajkoczy P. Distinct clinical and radiographic characteristics of moyamoya disease amongst European Caucasians. Eur J Neurol 2015;22:1012-1017.

48. Kaku Y, Morioka M, Ohmori Y, Kawano T, Kai Y, Fukuoka H, et al. Outer-diameter narrowing of the internal carotid and middle cerebral arteries in moyamoya disease detected on 3D constructive interference in steady-state MR image: is arterial constrictive remodeling a major pathogenesis? Acta Neurochir (Wien) 2012;154:2151-2157.

49. Yuan M, Liu ZQ, Wang ZQ, Li B, Xu LJ, Xiao XL. High-resolution MR imaging of the arterial wall in moyamoya disease. Neurosci Lett 2015;584:77-82.

50. Ryoo S, Cha J, Kim SJ, Choi JW, Ki CS, Kim KH, et al. Highresolution magnetic resonance wall imaging findings of moyamoya disease. Stroke 2014;45:2457-2460. 